PHYSICAL REVIEW E 71, 041501 (2005)

\title{
Electro-osmotic flow of a model electrolyte
}

\author{
Wei Zhu and Sherwin J. Singer* \\ Department of Chemistry, Ohio State University, Columbus, Ohio 43210, USA \\ Zhi Zheng \\ Biomedical Engineering Program, Ohio State University, Columbus, Ohio 43210, USA \\ A. T. Conlisk ${ }^{\dagger}$ \\ Department of Mechanical Engineering, Ohio State University, Columbus, Ohio 43210, USA
}

(Received 20 June 2004; published 8 April 2005)

\begin{abstract}
Electro-osmotic flow is studied by nonequilibrium molecular dynamics simulations in a model system chosen to elucidate various factors affecting the velocity profile and facilitate comparison with existing continuum theories. The model system consists of spherical ions and solvent, with stationary, uniformly charged walls that make a channel with a height of 20 particle diameters. We find that hydrodynamic theory adequately describes simple pressure-driven (Poiseuille) flow in this model. However, Poisson-Boltzmann theory fails to describe the ion distribution in important situations, and therefore continuum fluid dynamics based on the Poisson-Boltzmann ion distribution disagrees with simulation results in those situations. The failure of Poisson-Boltzmann theory is traced to the exclusion of ions near the channel walls resulting from reduced solvation of the ions in that region. When a corrected ion distribution is used as input for hydrodynamic theory, agreement with numerical simulations is restored. An analytic theory is presented that demonstrates that repulsion of the ions from the channel walls increases the flow rate, and attraction to the walls has the opposite effect. A recent numerical study of electro-osmotic flow is reanalyzed in the light of our findings, and the results conform well to our conclusions for the model system.
\end{abstract}

DOI: 10.1103/PhysRevE.71.041501

PACS number(s): 61.46.+w, 47.60.+i

\section{INTRODUCTION}

When chemically functionalized walls of a channel carry a net charge, electrolyte entering the channel acquires a compensating charge. Fluid flow can thereby be induced with applied electric fields. Electro-osmotic flow, that is, flow driven by applying an electric field to a charged fluid, has proved to be a useful technique for achieving fluid flow in a controlled manner on the nanometer scale, which is required in a variety of new technologies, such as fuel cells and micro- and nanoelectromechanical systems. These emerging technologies have spurred experimental and theoretical studies aimed at understanding field-driven flow of electrolytes in terms of fluid properties and the chemical properties of the channel walls. A common issue is repeatedly raised in these studies: Does hydrodynamic theory apply at the nanoscale? Two computational studies have recently appeared $[1,2]$ and they seem to answer this question in the negative, at least for "standard" hydrodynamic theory with a spatially uniform, linear constitutive relation between shear stress and strain rate. The SPC/E (extended simple point change) model for water, a three-site interaction model developed by Berendsen, Grigera, and Straatsma [3] was used in both studies, and channel walls were constructed from an array of Lennard-Jones particles. These models incorporate significant molecular detail, but of course the level of detail was

\footnotetext{
*Electronic address: singer@chemistry.ohio-state.edu

†Electronic address: conlisk.1@osu.edu
}

limited at some point to make the computations tractable. However, even modeling at this level poses a challenge to qualitative understanding, mainly because of the complexities of the polar, hydrogen-bonded aqueous solvent.

In this work we study a simplified electrolyte model to delve further into the issue of the applicability of hydrodynamic theory at the nanoscale. Both the ions and solvent are spherical, nonpolar particles interacting via Lennard-Jones pair potentials. Use of a simplified model is justified for the purpose of testing continuum theories, has precedent in several areas of condensed phase simulation, and can provide qualitative conclusions that should be applicable to real systems. Since continuum theory ignores molecular details, a simplified model involving spherical solvent particles is adequate for testing such theories. With this simplification, the simulations become more tractable while still retaining a model with discrete solvent particles. Indeed, replacing water molecules with spherical particles to gain computational convenience and theoretical simplicity has been done in other areas. For example, Klein and co-workers have recently developed a series of "coarse grain" models of biological systems where biomolecules are simplified and water molecules are replaced with spherical "W" particles [4-6], and there are earlier instances where simplified models have been used to deepen understanding of complex biological systems [7-9]. In other areas of statistical physics simple models have provided insights that have applied quite generally. For example, long time tails of the velocity autocorrelation function were first discovered by Alder and Wainwright in their studies of hard spheres and hard disks [10-12]. A study of 
electro-osmotic flow in this spirit by Thompson has recently appeared [13].

Our simplified model is chosen to be appropriate for comparison with existing continuum theory for electro-osmotic flow. To date, continuum theory for electro-osmotic flow has coupled Poisson-Boltzmann theory for the ionic distribution with a Navier-Stokes description of fluid flow. However, comparison of this theory with numerical simulations of ions in a confined polar fluid is not entirely appropriate. Ions in a polar fluid near a nonpolar wall experience decreased dielectric response of the polar fluid when near the interface. This effect is present in the molecular dynamics simulations using a polar solvent, and on the continuum level would be incorporated through image charges. Ordinary Poisson-Boltzmann theory does not include image effects, although image charges have been introduced in extensions of PoissonBoltzmann theory and more sophistical statistical mechanical treatments of the double layer [14-20]. Hence, the simplified model we simulate is more appropriate than more realistic models of an aqueous solvent for comparison with existing continuum theories of electro-osmotic flow. Also, because of its simplified nature, extensive simulations are possible and interpretation is less complicated. We are able to study an electrolyte containing both cations and anions with concentrations typical of experiments, whereas previous simulations included only ions of just one charge $[1,2]$. The physical properties of the Lennard-Jones fluid, including the viscosity $[21,22]$, has been very well characterized, aiding the comparison with hydrodynamic theory. Even though the solvent is nonpolar, strong short range solvation forces are still required to keep ions in solution. We show that these solvation forces strongly affect electro-osmotic flow because they affect the ion distribution, an effect that can be understood with a simple qualitative theory. We then apply our findings to reanalyze previous numerical work on electro-osmotic flow [2]. We determine that "standard" hydrodynamic theory, when appropriately applied as guided by our findings for the model system, accurately fits those results.

Electro-osmotic flow has been examined theoretically on many occasions in the past. The channel width is generally assumed to be much larger than the Debye screening length of the electrolyte. Under these conditions, most of the fluid in the channel can be treated as an ordinary, uncharged fluid [23]. Since electrokinetic effects are confined to a small region near the charged walls, a narrow region near each wall can be treated separately from other charged walls in the system (for example, the walls on the opposite side of the channel). Electro-osmotic phenomena in wide channels affect overall fluid flow by altering the hydrodynamic boundary conditions at the outer edge of the double layer.

In contrast, the channel dimensions are of the order of a Debye length for the case of nanochannel flow considered here, and inclusion of electro-osmotic effects does not simplify into a single-wall problem. Early treatments of overlapping double layers from walls within a Debye length of each other date back to the 1930s [24,25]. The first work on the electro-osmotic flow problem for overlapping double layers appears to have been done by Burgeen and Nakache [26]. They produced results for the velocity field and potential for two equally charged ions of valence $z$, assuming a Poisson-
Boltzmann distribution for the ions in solution. The convective terms in the velocity momentum equation were assumed to be negligible and the solution for the velocity and potential was assumed to be symmetric about the centerline of the channel. Additional work of a similar nature has also appeared [27-30]. Conlisk et al. [31] have provided a continuum theory for electro-osmotic flow valid not only in the small channel limit but also for large channels. Combining continuum ion transport equations with hydrodynamics for the electrolyte, they find that, under steady state conditions, the ion distribution is given by Poisson-Boltzmann theory and that the velocity flow profile is proportional to the difference in the electrostatic potential from its value at the walls [31].

Even though our solvent model is simplified, the conclusions we reach in this work should be applicable to real systems as long as they do not depend explicitly on the solvent molecular structure. The link we suggest between deviations of the ion density from Poisson-Boltzmann theory and flow rate is one such conclusion. By studying a range of concentrations and interactions strengths, we extract qualitative trends that were not apparent from earlier simulations. As discussed in our concluding section, this link seems to explain simulations of other, more realistic, models. Of course, there is also great value in very realistic models with high levels of molecular detail. We feel that progress is made by relating trends extracted from qualitative models to more detailed models and to experiment.

In Sec. II we define the model system. Results for large and small ionic interactions, compared to other interactions in the fluid, are presented in Sec. III. In Sec. IV we confirm that our simulations fall in the regime where the steady state velocity depends linearly on the driving field. Finally, we conclude by analyzing previous work and discussing some of the implications of our study.

\section{THE MODEL SYSTEM}

All particles of our nanochannel flow model interact via the Lennard-Jones potential

$$
v(r)=4 \epsilon\left[\left(\frac{\sigma}{r}\right)^{12}-\left(\frac{\sigma}{r}\right)^{6}\right] .
$$

In addition, there are Coulomb interactions between ions. For the most part we will use standard Lennard-Jones reduced units in this paper, measuring length in units of $\sigma$ and energy in units of $\epsilon$. Physical quantities are reduced by the $\epsilon$ and $\sigma$ parameters of the solvent-solvent interactions. Reduced quantities are indicated with an asterisk. For example, the solvent-solvent interaction in reduced units is $v^{*}\left(r^{*}\right)$ $=4\left[\left(r^{*}\right)^{-12}-\left(r^{*}\right)^{-6}\right]$. The walls are a single triangular closepacked layer of Lennard-Jones particles with a nearest neighbor distance of $1.462 \sigma$. The wall particles are identical to solvent except for being stationary (Fig. 1). In all cases, the wall-fluid particle Lennard-Jones parameters are the same as the fluid-fluid parameters. Adding further wall layers beyond the first layer would add an overall cohesive background potential, as it has been shown that the $r^{-12}$ repulsive poten- 


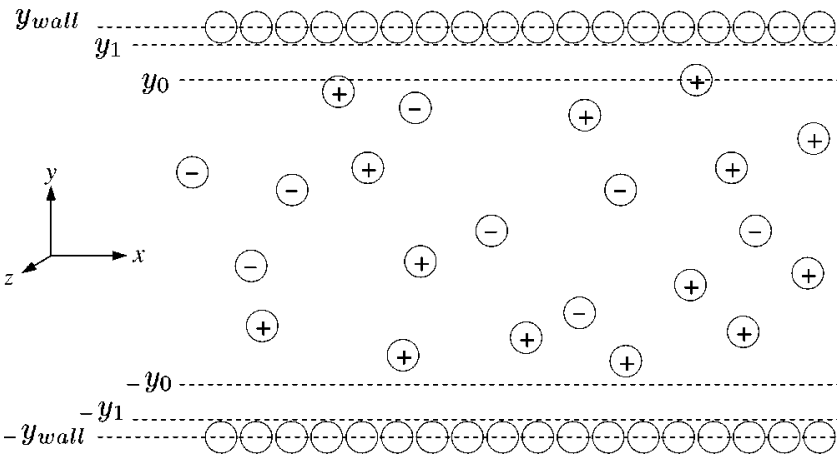

FIG. 1. Schematic depiction of the simulation cell. For clarity, only wall particles and ions, but not solvent, are shown. Periodic boundary conditions are enforced in the $x$ and $z$ directions. Closepacked layers of stationary wall particles are located at $y= \pm y_{\text {wall }}$. An effective volume for the fluid is defined by $y_{1}=y_{\text {wall }}-\sigma / 2$. Under some conditions, we find that ions are excluded from a region near the walls. We use $y_{0}$ to characterize the region available to the ions, and define $\Delta y_{\text {ion }}=y_{1}-y_{0}$.

tial from deeper layers is negligible and the $r^{-6}$ attraction from deeper layers is well approximated by a lateral average [32], which leads to a slowly varying attractive potential behaving like $r^{-3}$ at large separation. The fact that the wall particles are fixed might have some effect on our results. Sokhan et al. compared rigid and flexible walls in simulations of Lennard-Jones particles near a graphite surface [33] and within a carbon nanotube [34]. They found a large degree of slip, and observed that wall flexibility increased the fluid velocity at a graphite wall by $\sim 10 \%$.

The ions and wall particles have the same diameter as the solvent. All particles have the same mass $m_{\mathrm{LJ}}$. Strong ionsolvent attractions are needed to dissolve ions in solution. Since our solvent is nonpolar, we set $\epsilon_{\mathrm{IS}}^{*}$, the ion-solvent well depth in units of the solvent-solvent $\epsilon$, to a sufficiently large value to keep our ions from "precipitating" out of solution. The value of $\epsilon_{\mathrm{IS}}^{*}$ depended on the Coulomb interaction strength. Explicit values of $\epsilon_{\mathrm{IS}}^{*}$ are given in Table I.

The molarities in Table I were estimated on the basis of a particle diameter of $\sigma=2.8818 \AA$, which would make the total solution molarity equal to that of water, $55.5 \mathrm{M}$. The concentrations of ions were chosen to be those estimated in biomedical devices under development [35]. Assuming the same value of $\sigma$, the wall charge density in our system is $2.4 \times 10^{5}$ electron charges per $\mu \mathrm{m}^{2}$, which is considerably larger than the range of $(2-8) \times 10^{3}$ electron charges per $\mu \mathrm{m}^{2}$ recently estimated for silica glass [36]. Other workers simulated a system with just ions of a single charge, and overall solvent charge density of 2.7 [1] to 6 [2] times larger than ours. Their wall charge densities were 2.1 [1] or 3.1 [2] times larger.

The volume of the confined fluid is not well defined if the walls are soft. Following the procedure of Travis and Gubbins for Poiseuille flow of a Lennard-Jones fluid [37], we estimated an effective channel width by subtracting $\sigma$ from the distance between the wall particles on either side of the channel. We define $y_{1}$ as the effective distance that particles extend from the center of the channel. Throughout this work, we use $y_{1}^{*}=y_{\text {wall }}^{*}-\frac{1}{2}$ (Fig. 1). As shown below, this value of $y_{1}^{*}$ provides an excellent match to continuum theory for our system when tested for Poiseuille flow. Based on this volume estimate, the overall number density of the fluid particles, including both solvent and ions, was close to $\rho^{*}=\rho \sigma^{3}=0.8$ in all our simulations. As seen below in Figs. 3, 4, and 7, our channels are sufficiently wide so that a region with flat number density equal to the overall density is present at the center of the channel. The reduced temperature was $T^{*}=k_{B} T / \epsilon$ $=1$, which puts our model fluid in the liquid region of the Lennard-Jones phase diagram.

The Coulombic part of the interaction between ions $i$ and $j$ in reduced units is

$$
v_{C}^{*}\left(r_{i}^{*}-r_{j}^{*}\right)=\frac{e^{2}}{\epsilon_{r} \epsilon \sigma} \frac{z_{i} z_{j}}{\left|r_{i}^{*}-r_{j}^{*}\right|} \equiv \zeta \frac{z_{i} z_{j}}{\left|r_{i}^{*}-r_{j}^{*}\right|},
$$

where $e$ is the magnitude of the electron charge, $z_{i}$ and $z_{j}$ are the ionic charges measured in units of $e$, and $\epsilon_{r}$ is the dielectric constant of the medium. In reduced units, the ratio of the Coulomb to Lennard-Jones interaction energy is $\zeta$. The external electric field produced a constant force of

$$
z_{i} E^{*}
$$

on the $i$ th ion in the $x$ direction. The reduced electric field is

$$
E^{*}=\frac{\sigma e}{\epsilon} E,
$$

where $E$ is the magnitude of the electric field. The Coulomb energy and force were calculated using a two-dimensional Ewald sum [38,39]. We employed the method of Kawata and Mikami [40] to turn the reciprocal space portion of the twodimensional Ewald expression into a factorized form resembling the three-dimensional expression. This also removes the error function evaluations from the reciprocal space sum.

TABLE I. Summary of nonequilibrium molecular dynamics simulations performed. In all cases, the system was periodically replicated in the $x$ direction, the direction of flow, and the $z$ direction, with dimensions $L_{x}^{*}=21.77$ and $L_{z}^{*}=21.83$, respectively. In the $y$ direction, wall particles were placed at $y^{*}= \pm y_{\text {wall }}^{*}$ and the volume available to particles, used to calculate the ion molarities $(M)$ given in the rightmost columns, was estimated on the basis of an effective $y$ dimension of $L_{y}^{*}=2 y_{\text {wall }}^{*}-1$ and a fluid particle diameter of $2.8818 \AA$. The parameter $\zeta$ is defined in Eq. (2).

\begin{tabular}{ccccccccccc}
\hline \hline Run & Total & Cation & Anions & Solvent & Wall & $y_{\text {wall }}^{*}$ & $\zeta$ & $\epsilon_{\mathrm{IS}}^{*}$ & $M$ (cation) & $M($ anion $)$ \\
\hline $\mathrm{A}$ & 8638 & 31 & 12 & 7757 & 836 & 10.76 & 5 & 7 & 0.2206 & 0.08538 \\
$\mathrm{~B}$ & 8638 & 31 & 12 & 7757 & 836 & 10.76 & 1 & 1.4 & 0.2206 & 0.08538 \\
\hline \hline
\end{tabular}


Whereas Kawata and Mikami only perform this transformation for nonzero reciprocal lattice vectors, we were able to transform the zero reciprocal lattice vector term as well [41], thereby completely eliminating error function evaluations from the reciprocal space part of the Ewald energy and force.

In our simulations the $y$ direction is perpendicular to the walls. We induce flow in the $x$ direction. The simulation cell will rapidly heat up unless we remove energy from the system. We use the Gaussian isokinetic thermostat $[42,43]$ to maintain the system temperature. Zhang's integrator [44] is used to solve the non-Hamiltonian equations of motion. We used a time step of $\Delta t^{*}=0.01$, where the reduced unit of time is $\sqrt{m_{\mathrm{LJ}} / \epsilon} \sigma$. This value of $\Delta t^{*}$ yielded excellent preservation of the isokinetic conditions, which would be exactly conserved without round-off error and force discontinuities caused by the cutting off of the Lennard-Jones force at $2.5 \sigma$. The time step $\Delta t^{*}$ was chosen to be well within the range of acceptable values reported by Zhang for a Lennard-Jones system [44], and was verified to give converged particle positions after short (reduced time $\sim 1$ ) runs. Only the $y$ and $z$ components of the velocity are thermostated because there is a nonzero streaming velocity in the $x$ direction. In the $x$ direction, what has to be thermostated is the so-called "peculiar" velocity [43], the velocity relative to the streaming velocity. Therefore, thermostating the $x$ velocities in principle involves first finding the average or streaming velocity profile, although other workers have neglected the streaming velocity when it was small compared to the peculiar velocity $[1,2]$. When the flow velocity is comparable to the thermal velocity, it is more rigorous, and also avoids the necessity of tracking the peculiar velocity, to thermostat only the $y$ and $z$ directions. Heat flow between the $x$ motion and other (thermostated) degrees of freedom keeps the total system at constant temperature. We have verified that energy flow between the $x$ direction and the other two directions is very rapid.

Because of the qualitative differences between our model solvent and a realistic model of water, it is impossible to choose a value of $\zeta$ [Eq. (2)], the relative Coulomb interaction strength, that will approximately map the behavior of aqueous electrolytes onto the model system. For example, vastly different values of $\epsilon$ are inferred by a typical hydrogen-bond strength, or alternatively by choosing $\epsilon$ so that the Lennard-Jones solvent has triple and critical temperatures near those of water. Instead, we investigated two values of $\zeta, 1$ and 5, that we will see span the range from small to large interaction strengths.

We verified that hydrodynamics with no-slip boundary conditions enforced at $y_{1}^{*}$ provides a good description of ordinary Poiseuille flow in our model system. Figure 2 shows the density and velocity profile for a system with no ions, and a constant force $f^{*}=0.02$ on solvent particles. The velocity profile obtained from simulations is compared with the standard result for an incompressible fluid with overall number density $\rho^{*}$ and stick boundary conditions enforced at $y_{1}^{*}$ :

$$
v^{*}(y *)=\frac{f^{*} \rho^{*}}{2 \eta^{*}}\left[\left(y_{1}^{*}\right)^{2}-\left(y^{*}\right)^{2}\right] .
$$

Layering of the fluid near the walls is quite pronounced at the density and temperature of our simulations. Despite this

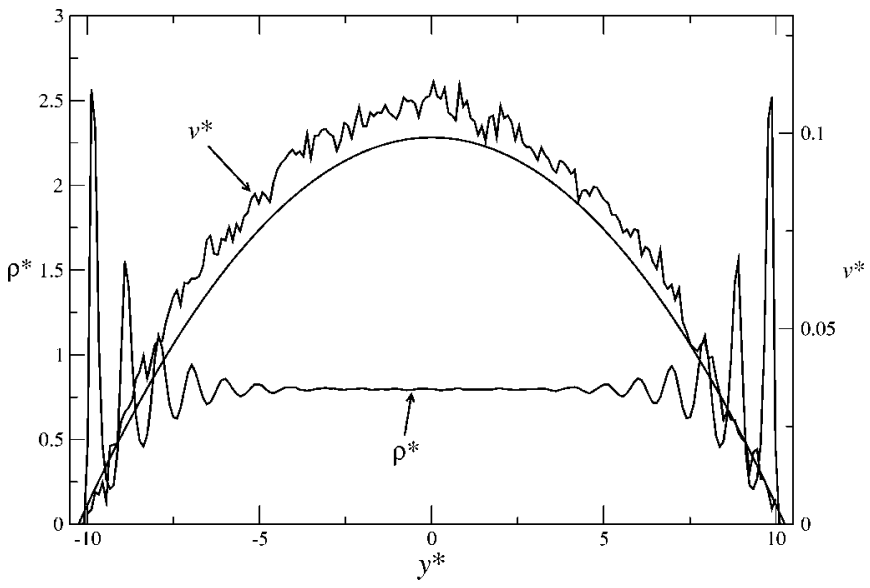

FIG. 2. Steady state density and velocity distributions established under conditions of planar Poiseuille flow for a pure Lennard-Jones fluid at $\rho^{*}=0.8, T^{*}=1.0$. Flow was induced by a constant force $f^{*}=0.005$ applied to all particles. The velocity profile from molecular dynamics is compared with hydrodynamic theory [Eq. (5)] for planar Poiseuille flow (smooth curve). The reduced viscosity used to calculate the continuum theory velocity profile was taken as $\eta^{*}=2.13$, reported for simulations of a pure LennardJones fluid [22] at $\rho^{*}=0.8, T^{*}=1.0$. Wall particles were located at $Y_{\text {wall }}^{*}= \pm 10.76$. We took $y_{1}^{*}=y_{\text {wall }}^{*}-1 / 2$ as the point where stick boundary conditions were enforced.

structure near the walls, continuum theory provides a quantitative description of the velocity profile in all regions of the channel, including near the walls. In Fig. 2 our velocity profile from simulations of Poiseuille flow is compared with the profile calculated from Eq. (5) with reduced viscosity $\eta^{*}$ $=2.13$, as reported [22] for simulations of a Lennard-Jones fluid at $\rho^{*}=0.8, T^{*}=1.0$, and $y_{1}^{*}=y_{\text {wall }}^{*}-\frac{1}{2}=10.26$. Almost the same values of $\eta^{*}$ and $y_{1}^{*}, 1.96$ and 10.28 , respectively, are obtained from a least squares fit to the data in Fig. 2. For Poiseuille flow in our system, continuum theory with stick boundary conditions imposed at $y_{1}^{*}=y_{\text {wall }}^{*}-\frac{1}{2}$ is evidently quite successful.

The validity of stick boundary conditions has been examined recently, through both simulations and experiment. Slip has been observed in simulations under a variety of conditions: nonwetting fluids [45,46], fluids with weak interactions with walls [47-49], low-density fluids [50-55], molecular fluids $[48,49,56]$, large shear rates [57], and fluids in which the corrugation length scale of the wall departs significantly from that of the fluid particles [58]. While a variety of conditions will generate slip, it is not clear that a significant amount of slip is a generic phenomenon. In the absence of the conditions listed above, very small slip lengths have been reported $[47,59]$. Gao et al. have shown that a rootmean-square wall roughness of 1.6 particle diameters was sufficient to suppress all evidence of slip in simulations of $n$-hexadecane [59]. For the Poiseuille flow conditions that gave rise to the data shown in Fig. 2, the corrugation wavelength of the walls is comparable to the fluid particle size and the interactions between fluid particles and fixed wall particles is identical to fluid-fluid particle interactions. Hence the absence of slip in this case is to be expected. Since these 

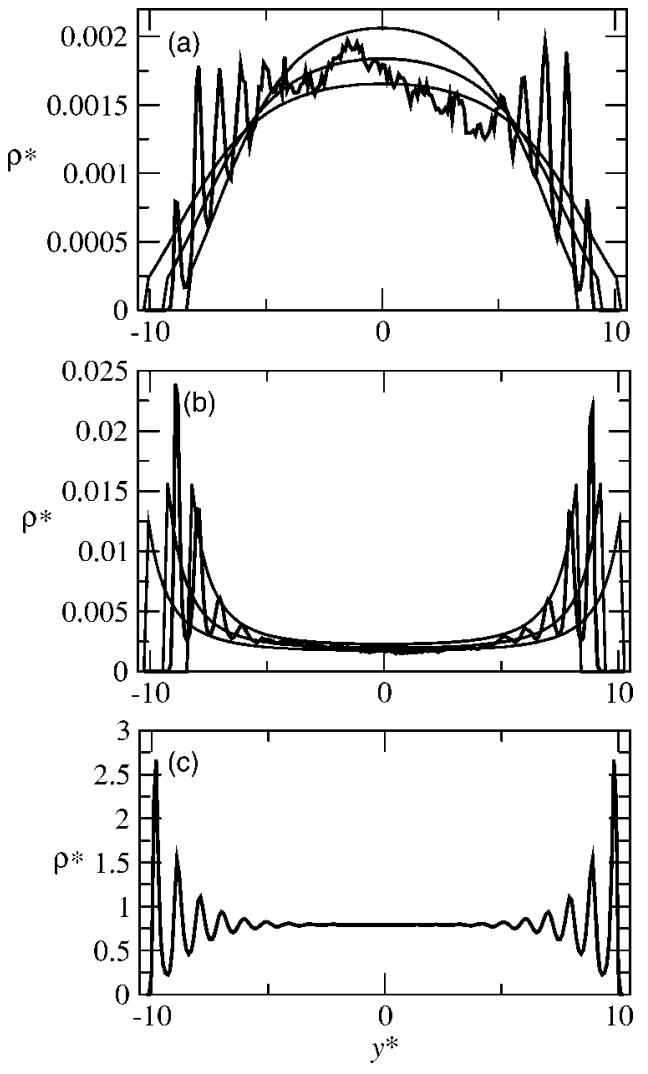

FIG. 3. Density of (a) anions, (b) cations, and (c) solvent as a function of position between the channel walls for the simulation conditions A described in Table I. The fluid is subjected to an electric field $E^{*}=2$. The smooth curves in panels (a) and (b) are the ion distributions predicted by Poisson-Boltzmann theory in which the ions are confined to the region $\left|y^{*}\right|<y_{1}^{*}-\Delta y_{\text {ion }}^{*}$, with $\Delta y_{\text {ion }}^{*}=0,1,2$. The curves corresponding to different values of $\Delta y_{\text {ion }}^{*}$ are distinguished by the $y$ value at which the densities fall to zero.

conditions also apply to our simulation of electro-osmotic flow, slip is not observed in the simulations reported in later sections. Several studies have demonstrated the breakdown of hydrodynamics with a linear shear-stress-strain-rate relation in very narrow channels, channels in the range of $\sim 5$ fluid particle diameters $[2,37,56,60,61]$. Currently, electroosmotic flow devices are not commonly fabricated with these very small channel widths [62-65], and therefore we did not probe this regime in this study.

\section{RESULTS OF NONEQUILIBRIUM MOLECULAR DYNAMICS SIMULATIONS}

We chose simulation conditions in which the ion concentrations (see Table I) are typical of experimental conditions. The total number of ions in the simulation cell was consequently quite low. The small number of ions required sufficiently long simulations, typically on the order of $10^{6}$ times steps with the longest being $\sim 2.5 \times 10^{6}$ steps, to gather reliable statistics. Other workers simulated a system with just ions of a single charge, and 2.7 to 6 times larger ionic concentrations $[1,2]$.
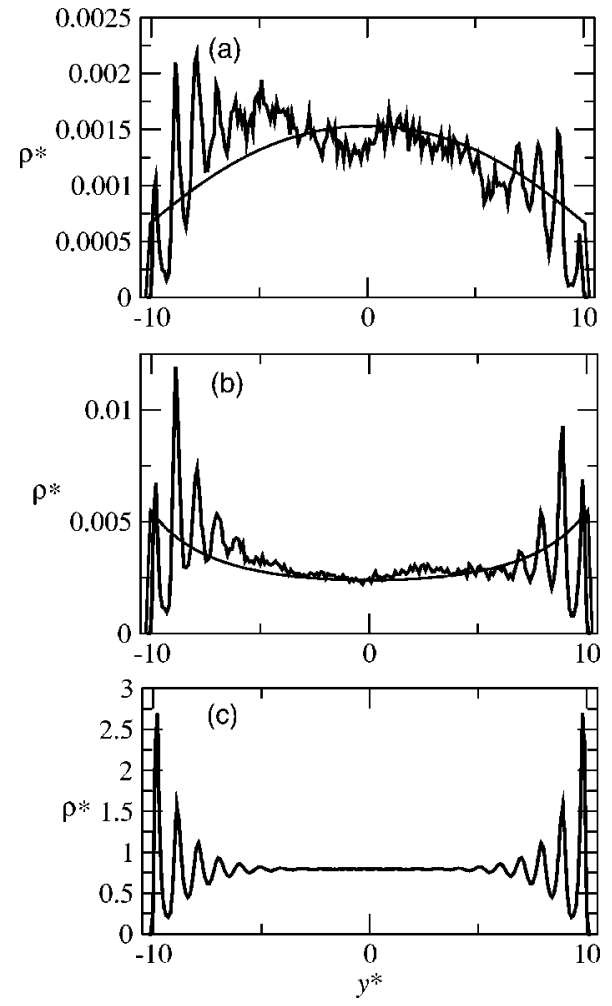

FIG. 4. Density of (a) anions, (b) cations, and (c) solvent as a function of position between the channel walls for the simulation conditions B described in Table I. The fluid is subjected to an electric field $E^{*}=2$. The ion distribution predicted by PoissonBoltzmann theory is also shown.

The density profiles from runs A and B, as shown in Figs. 3 and 4, respectively, are qualitatively different. The Coulomb interaction strength $\zeta$ is five times larger in run A compared to run $\mathrm{B}$, and consequently the ion-solvent interaction strength $\epsilon_{\mathrm{IS}}^{*}$ is much larger as well to maintain ions in solution (see Table I). Ions layer directly against the walls in run $\mathrm{B}$, but they are completely absent from the wall layer in run A. The different behavior near the walls originates from the strong ion-solvent interactions in run A. For an ion to enter the wall layer, it must shed several of its strongly stabilizing solvent neighbors. Hence, under conditions with strong ionion and ion-solvent interactions, as in run A, there is a significant energetic barrier for ions to enter the layer of fluid adjacent to the wall. The ion-solvent attractions in run B are sufficiently reduced to allow ions in under these conditions to enter the wall layer without significant energetic penalty. However, a mild depletion of ions from the wall region still occurs for run B, as evidenced by the outermost peak in the cation density being smaller than the adjacent peak in Fig. 4(b).

As mentioned in the Introduction, in a polar solvent exclusion of ions from an interface with a medium of low dielectric constant is a well known phenomenon. The prototypical example is depletion of ions near an air-water interface [15]. Hence, it is a realistic possibility in some fabricated devices that ions may be excluded from nanochannel walls, depending on the chemical nature of the ions and functionalization of the walls. In our model system, the 

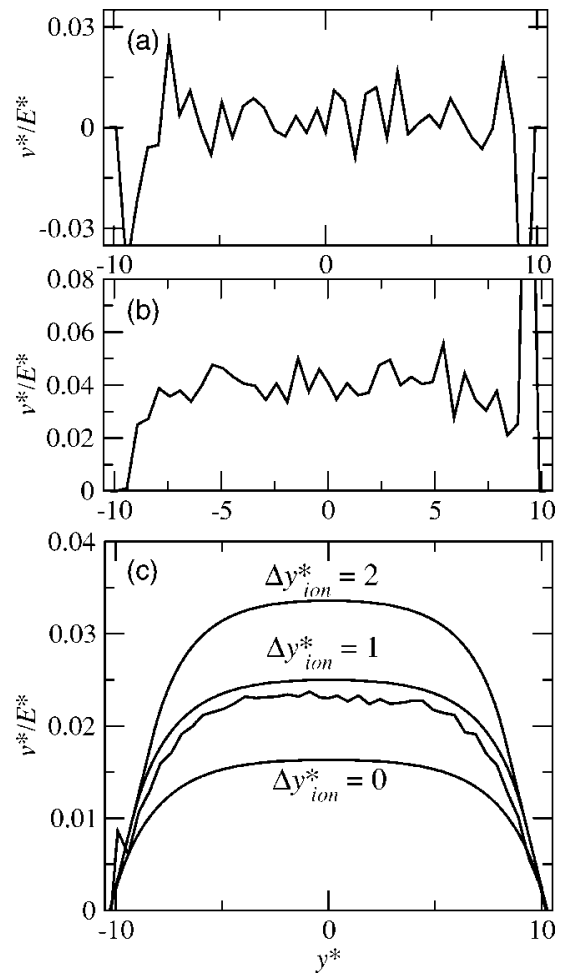

FIG. 5. Velocity distribution divided by the reduced field strength $E^{*}$ for (a) anions, (b) cations, and (c) solvent as a function of position between the channel walls for the simulation conditions A described in Table I. The fluid is subjected to an electric field $E^{*}=2$. In panel (c), molecular dynamics simulation results are compared with the velocity profile of Eq. (16) calculated with different values of $\Delta y_{\text {ion }}^{*}$ [Eq. (6)] as indicated in the figure.

simulation conditions A and B portray opposite regimes regarding the importance of ion exclusion from the interface. In our model system the effects are short range and confined to the layer adjacent to the wall. In a more realistic model, the exclusion would be described by long range repulsions from image charges.

In Figs. 3 and 4 the simulation results are compared with Poisson-Boltzmann theory. The Debye length $\kappa^{-1}$ [see Eq. (A6)] of Poisson-Boltzmann theory was $2.16 \sigma$ and $4.56 \sigma$ for the conditions of runs A and B, Figs. 3 and 4, respectively. The solution of the Poisson-Boltzmann equations is reviewed in the Appendix. Here we briefly mention that to relate the continuum theory to molecular dynamics simulations, only the total number of cations and anions in the channel, and the channel width accessible to ions $\left[\left|y^{*}\right|<y_{1}^{*}\right.$ with $y_{1}^{*}=y_{\text {wall }}^{*}-\frac{1}{2}$ (see Fig. 1)] must be specified. Poisson-Boltzmann theory gives a good representation of the ionic profile away from the walls in run B, and the density from simulations seems to oscillate about an average position described by the PoissonBoltzmann theory in the region of strong layering near the walls.

The agreement between Poisson-Boltzmann theory (the smooth curves in Fig. 3 for $\Delta y_{\text {ion }}^{*}=0$ ) and simulations is not even qualitatively correct in run A because the continuum theory does not describe ion exclusion from the walls. Although this effect arises from solvation forces, we can qualitatively incorporate the effect with a modified Poisson-
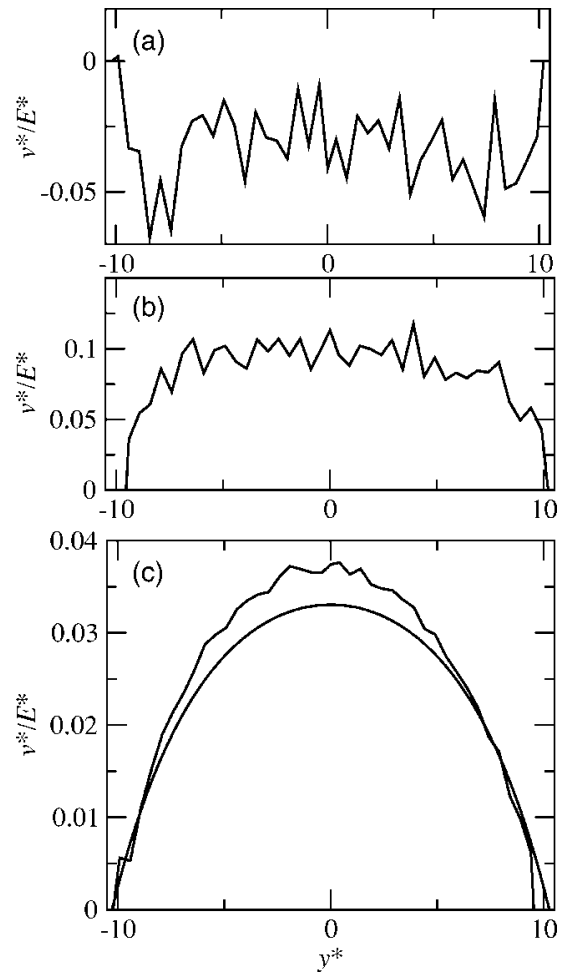

FIG. 6. Velocity distribution divided by the reduced field strength $E^{*}$ for (a) anions, (b) cations, and (c) solvent as a function of position between the channel walls for the simulation conditions $\mathrm{B}$ described in Table I. The fluid is subjected to an electric field $E^{*}=2$.

Boltzmann theory by letting $y_{0}$, the distance ions extend from the center of the channel, depart from $y_{1}$, which we continue to use to define the volume accessible to the solvent (Fig. 1). We define $\Delta y_{\text {ion }}$ to be the width of the exclusion region.

$$
\Delta y_{\text {ion }} \equiv y_{1}-y_{0} .
$$

In Fig. 3, the distribution of ions in run $\mathrm{A}$ is compared with Poisson-Boltzmann theory in which $\Delta y_{\text {ion }}^{*}$ is set equal to 0,1 , or 2. None of the modifications yield continuum distributions in quantitative agreement with simulations, even if the density oscillations near the walls are smoothed. However, it is clear that confining the ions to a smaller volume does improve the qualitative agreement between simulations and continuum theory, and that reducing the distance of closest approach by $\Delta y_{\text {ion }}^{*}=1$ on either side of the channel produces the best agreement for the anion density, and for the cation density everywhere except close to the walls. Below, we will demonstrate that these modifications bring the continuum theory velocity profile in qualitative agreement with simulations.

Velocity profiles for ions and solvent are exhibited in Fig. 5 for run A and Fig. 6 for run B. In both cases the cations flow substantially faster than the solvent, while the anions, which are pulled by the electric field against the flow, have an average velocity close to zero in run A or even negative in run $\mathrm{B}$. The continuum prediction for the flow profile is obtained from the Navier-Stokes equation with force density 
given by $q(\mathbf{r}) \mathbf{E}$, where $\mathbf{E}$ is the electric field. Specializing to fully developed flow of an incompressible fluid, the $x$-momentum Navier-Stokes equation reduces exactly to the Stokes equation,

$$
\frac{\partial^{2} v_{x}}{\partial y^{2}}=-\frac{q(y) E}{\eta} .
$$

As noted by Conlisk et al. [31], Eq. (7) for $v_{x}(y)$ is identical to the Poisson-Boltzmann equation (A5) for the electrostatic potential $\phi(y)$ to within a constant factor. (This conclusion is also implicit in equations for electro-osmotic flow developed earlier, for example by Cummings et al. [23].) Consequently, the velocity profile is related to the electrostatic potential as

$$
v_{x}(y)=\frac{\epsilon_{r} E}{4 \pi \eta}\left[\phi(y)-\phi\left(y_{1}\right)\right]
$$

when both the ion density and velocity distribution are assumed to vanish at $y_{1}$. In terms of reduced quantities, including the reduced electrostatic potential $\psi=\left(z e / k_{B} T\right) \phi$,

$$
v_{x}^{*}\left(y^{*}\right)=\frac{E^{*} T^{*}}{4 \pi z \zeta \eta^{*}}\left[\psi\left(y^{*}\right)-\psi\left(y_{1}^{*}\right)\right] .
$$

Comparison of continuum theory velocity profiles [Eq. (9)] with simulation results is made in Figs. 5 and 6. There is only a slight disagreement for the conditions of run B (Fig. 6 ), where the smaller ion-solvent interactions do not alter the ion distribution radically from the Poisson-Boltzmann result. However, in run A the steady state solvent velocity in molecular dynamics simulations exceeds the prediction of continuum theory by roughly $50 \%$. The question arises as to whether the entire continuum approach fails for this situation, or whether the disagreement in the velocity profile is the result of ion exclusion from the wall region previously noted for run A. In our discussion of the ion density distribution, we qualitatively modeled the effect of ion exclusion from the wall region by solving the Poisson-Boltzmann equations for ions confined to a region smaller than what would be expected from the hard core repulsions between ions and the wall particles. Here we consider the velocity profile expected from that modified Poisson-Boltzmann theory. The Navier-Stokes equation (7) can be easily solved for the modified charge distribution that goes to zero at $y_{0}$, which is smaller than the distance $y_{1}$ at which stick boundary conditions are imposed on $v_{x}(y)$. In the region $|y|$ $<y_{0}, v_{x}(y)$ and the electrostatic potential $\phi(y)$ satisfy, within constant factors, the same second order differential equation, and are linearly related to each other. For $y_{0}<|y|<y_{1}$, the velocity profile satisfies Eq. (7) in which $q(y)=0$. Therefore the entire velocity profile is given by

$$
v_{x}(y)= \begin{cases}\frac{\epsilon_{r} E}{4 \pi \eta}\left[\phi(y)-\phi\left(y_{0}\right)\right]+v_{x}\left(y_{0}\right), & |y|<y_{0}, \\ v_{x}^{\prime}\left(y_{0}\right)\left(|y|-y_{1}\right), & y_{0}<|y|<y_{1},\end{cases}
$$

where $v_{x}^{\prime}\left(y_{0}\right)$ is the $y$ derivative of $v_{x}(y)$ at $y_{0}$.

We can derive an expression for $v_{x}^{\prime}\left(y_{0}\right)$ using

$$
v_{x}^{\prime}(y)=\frac{\epsilon_{r} E}{4 \pi \eta} \phi^{\prime}(y)
$$

and invoking standard electrostatic boundary conditions

$$
\frac{\epsilon_{r}}{4 \pi} \phi^{\prime}\left(y_{0}\right)=\Sigma(\text { wall charge density })=-y_{0} q .
$$

In the above equation, $q$ is the overall charge density, and the second equality follows from overall charge neutrality. Combining the previous two equations, we find

$$
v^{\prime}\left(y_{0}\right)=-\frac{q E y_{0}}{\eta} .
$$

Now that we have the derivative $v_{x}^{\prime}\left(y_{0}\right)$ in hand, we can obtain an expression for the value of the velocity at $y_{0}$,

$$
v_{x}\left(y_{0}\right)=v_{x}^{\prime}\left(y_{0}\right)\left(y_{0}-y_{1}\right)=\frac{q E y_{0}\left(y_{1}-y_{0}\right)}{\eta},
$$

and throughout the excluded region,

$$
v_{x}(y)=\frac{q E y_{0}}{\eta}\left(y_{1}-|y|\right), \quad y_{0}<|y|<y_{1} .
$$

Finally, we can set the whole solution in reduced units:

$$
v_{x}^{*}\left(y^{*}\right)= \begin{cases}\frac{E^{*} T^{*}}{4 \pi z \zeta \eta^{*}}\left[\psi\left(y^{*}\right)-\psi\left(y_{0}^{*}\right)\right]+v_{x}^{*}\left(y_{0}^{*}\right), & \left|y^{*}\right|<y_{0}^{*}, \\ v_{x}^{*}\left(y_{0}^{*}\right)\left(\frac{y_{1}^{*}-\left|y^{*}\right|}{y_{1}^{*}-y_{0}^{*}}\right), & y_{0}^{*}<\left|y^{*}\right|<y_{1}^{*},\end{cases}
$$

where

$$
v_{x}^{*}\left(y_{0}^{*}\right)=\frac{q^{*} E^{*} y_{0}^{*}\left(y_{1}^{*}-y_{0}^{*}\right)}{\eta^{*}} .
$$

The solvent velocity profile predicted by Eq. (16) is compared with molecular dynamics results in Fig. 5. The series of continuum theory curves with different values of $\Delta y_{\text {ion }}$ confirm that exclusion of ions from the wall region increases flow in response to an applied field. In fact, the velocity profile for $\Delta y_{\mathrm{ion}}^{*}=1$ is in best agreement with simulations. The fact that the simulated velocity profile is slightly above the continuum theory profile for run B (Fig. 6) can also be traced to the small amount of ion exclusion near the walls in run $\mathrm{B}$. In the following section, more evidence is presented that corrections to continuum theory enter principally through the charge density. The theory presented in this section predicts a qualitative trend that should be useful in the design of nanochannels: shifting the charge density toward the center of the channel increases the flow rate, while a shift toward the walls decreases the flow. 

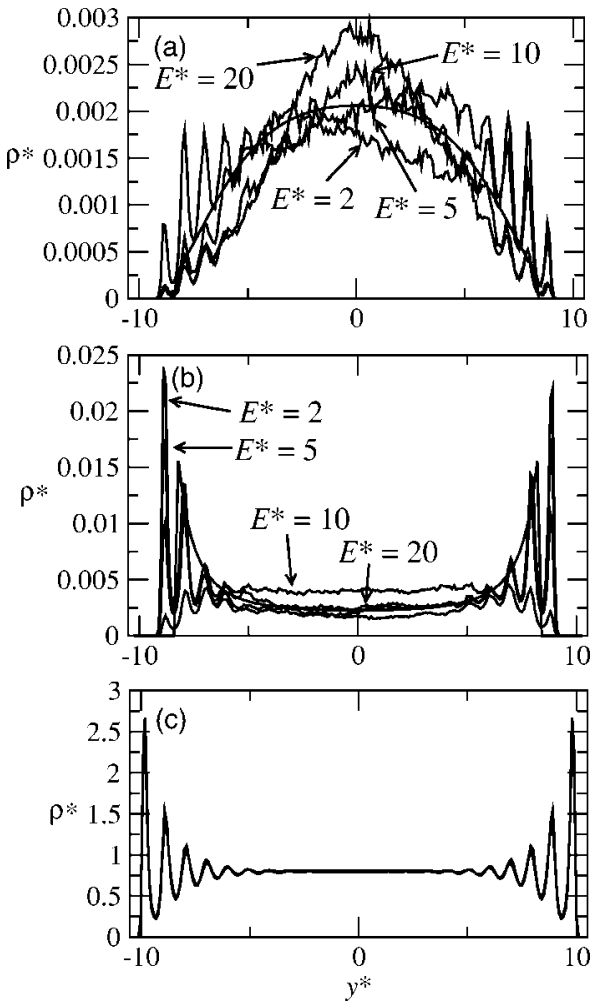

FIG. 7. Density of (a) anions, (b) cations, and (c) solvent as a function of position between the channel walls for the simulation conditions A described in Table I. Results are presented for electric field strengths $E^{*}=2,5,10$, and 20. With increasing field strength and flow rate, the ions move progressively toward the center of the channel. The ion densities for $E^{*}=10$ are seen to match well with the ion densities for the modified Poisson-Boltzmann theory with $\Delta y_{\text {ion }}^{*}=2$, which is the smooth curve plotted in panels (a) and (b). The solvent density is virtually independent of applied field and flow rate, so the various curves in panel (c) are not labeled.

\section{LINEAR AND NONLINEAR FLOW RESPONSE}

Our field strengths are chosen to be quite large compared to real experiments in order to enhance the signal-to-noise ratio in the accumulation of velocity profiles. As reported in this section, care was taken to verify that data reported in Figs. 5 and 6 are in the regime dominated by linear response. At larger field strength, we observed pronounced nonlinearity in the flow response. The fields at which nonlinearity sets in are quite large, as are the induced velocities. The induced flow was $10 \%$ or less of a typical thermal velocity for the conditions discussed in the previous section. A series of trials at varying driving field $E^{*}$ presented here allows us to estimate how close the data of the previous section are to the linear response limit.

In Fig. 7 the density of ions and solvent is shown for several values of field strength $E^{*}$. As the driving force on the ions is increased, they leave the slow-moving region near the walls in favor of the central part of the channel where the flow rate is larger. The $v^{*} / E^{*}$ data presented in Fig. 8 indicates that the flow response increases supralinearly when large fields are applied. The maximum of the velocity profile at the center of the channel can be fitted to the form
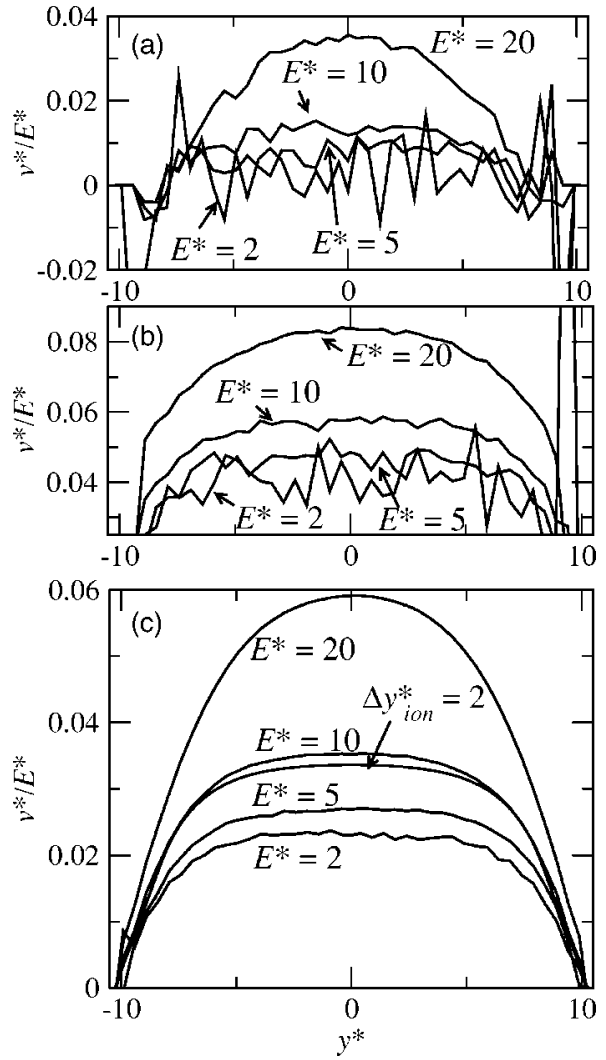

FIG. 8. Velocity distribution divided by the reduced field strength $E^{*}$ for (a) anions, (b) cations, and (c) solvent as a function of position between the channel walls for the simulation conditions A described in Table I. Results are presented for electric fields strengths $E^{*}=2,5,10$, and 20 . The flow rate has a supralinear dependence on field strength for high fields. (Note that $v^{*} / E^{*}$ is plotted.) The solvent velocity profile for $E^{*}=10$ is seen to match well with the continuum velocity profile calculated from the modified Poisson-Boltzmann theory with $\Delta y_{\text {ion }}^{*}=2$, which is the smooth curve plotted in panel (c).

$$
\left.\frac{v^{*}}{E^{*}}\right|_{y^{*}=0}=0.0223+6.83 \times 10^{-4} E^{*}+5.760 \times 10^{-5}\left(E^{*}\right)^{2} .
$$

This relation permits an extrapolation of $v^{*} / E^{*}$ to the limit of vanishing field $E^{*}$. The ratio $\left(v^{*} / E^{*}\right)_{y^{*}=0}$ reported for $E^{*}=2$ in Fig. 5 is 0.024 . The value departs by roughly $7 \%$ from its extrapolation to zero field strength, 0.0223 , according to the fit in Eq. (18).

The theory introduced in the previous section shows that the flow rate increases when ions are shifted away from the wall region. In fact, the supralinear high-field artifacts arise from the shift in ion density depicted in Fig. 7. After correction for the shifted density, the hydrodynamic equations still apply in this regime. In Fig. 7 the modified PoissonBoltzmann ion density for $\Delta y_{\text {ion }}^{*}=2$ qualitatively matches the simulation results for $E^{*}=10$. The modified PoissonBoltzmann theory tracks the enhanced density near the center of the channel, and follows the average of the density oscillations near the walls. The close agreement of the veloc- 
ity profile arising from $\Delta y_{\text {ion }}^{*}=2$ with simulation results for $E^{*}=10$ in Fig. 8 confirms that correction of the ion density is the major nonlinear effect.

\section{DISCUSSION}

Electro-osmotic flow of an aqueous electrolyte has been examined in two recent simulation studies $[1,2]$. In these studies, the ion distribution obtained from simulations disagreed with Poisson-Boltzmann theory. An attempt was made to extract the shear stress and strain rate as a function of position in the channel, and to test the validity of a linear constitutive relation. In both studies, the the linear constitutive relation was judged to fail because the viscosity was a function of position within the channel, and found to rise steeply near the walls. Qiao and Aluru [2] concluded that continuum theory with a spatially uniform, linear constitutive relation between shear stress and strain, even when corrected for the failure of Poisson-Boltzmann theory to describe the ion distribution, still gave a velocity profile more than twice that of simulations. They traced this error to the departure of the viscosity from the bulk liquid value near the channel walls. In this section we will discuss these results in the light of the findings presented in this paper.

Several issues must be discussed in relation to tests made by Freund [1] and Qiao and Aluru [2] of the linear relation between shear stress and strain. We have verified that such tests, which involve integrating the charge density and differentiation of the velocity profile, are very sensitive to the manner in which numerical data from simulations is treated. Freund first smoothed the velocity profile, and then fitted the profile to a combined exponential ansatz and Fourier series. He found that the ratio of shear stress to strain rate was rather close to the experimental dynamical viscosity of water through most of the channel. An increase in the effective viscosity was observed within $10 \AA$ of the channel walls. Regardless of the numerical treatment of the simulation data to obtain a viscosity profile, there is a clear rise in viscosity near the walls. However, the extent to which hydrodynamic predictions are affected by increased viscosity in this narrow region where velocity is near zero is not clear.

Our analysis in Sec. III using the modified PoissonBoltzmann theory can be regarded as a similar attempt to fit shear stress and strain rate from simulations, and test the validity of a linear constitutive relation. Although we never attempted to apply the modified Poisson-Boltzmann theory as a least squares fit, the success with which we can simultaneously describe the charge densities and velocity profiles with the modified Poisson-Boltzmann theory indicates that linear hydrodynamics with constant viscosity performs adequately for our model system, provided that an accurate charge density is input into the hydrodynamic theory. In our analysis, the stress-strain ratio was fixed at the reported viscosity for a bulk Lennard-Jones fluid. Indeed, there may be local variations in this ratio, especially near the walls, as found by Freund [1], even though neglect of these local variations in our analytic theory evidently does not spoil the overall predicted velocity profile. Pronounced charge density oscillations near the walls in our system, much stronger than

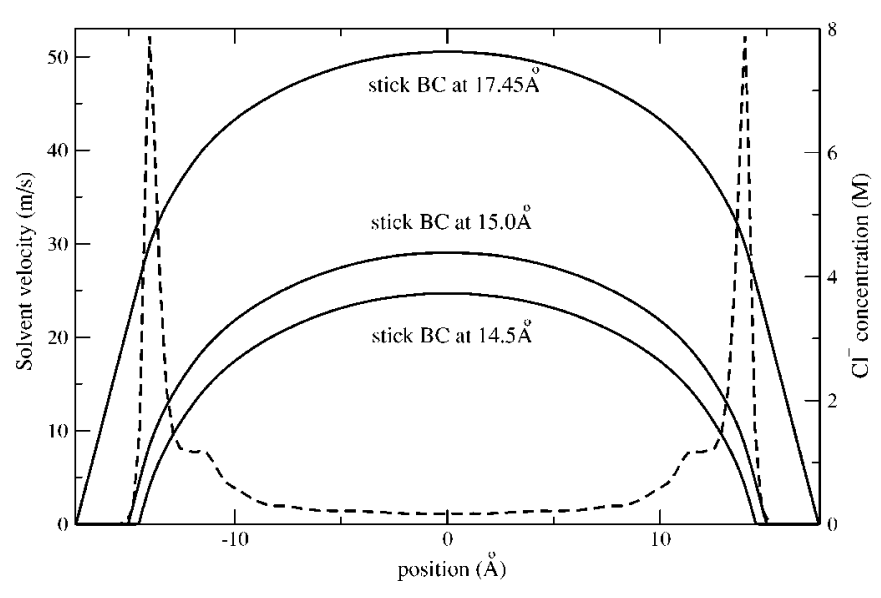

FIG. 9. The solid curves are the velocity distributions for the case 1 system studied by Qiao and Aluru [2]. The three steady state velocity profiles were calculated using the Navier-Stokes equation with stick boundary conditions (BC) enforced at three different surfaces. The distance of the boundary surface from the channel center is indicated in the figure. The charge density used in the hydrodynamic calculations was the chloride ion distribution from the simulations of Qiao and Aluru kindly supplied to us by the authors, and shown as the dashed line in the figure. There was only one type of ion in these simulations. The parameters employed for the fluid in the continuum calculations were $T=300 \mathrm{~K}, \epsilon_{r}=81$, and $\eta=7.43$ $\times 10^{-4} \mathrm{Pas}$. We use the viscosity determined for their system by Qiao and Aluru [2], which is higher than the viscosity 6.6 $\times 10^{-4} \mathrm{~Pa}$ s reported for pure SPC/E water [67] because of the somewhat high concentration of ions used in those simulations.

the oscillations reported in the simulations of the SPC/E water model, prevented us from completing a detailed analysis of the effective viscosity near the walls. Continuum theory using the bulk Lennard-Jones liquid viscosity provided an accurate description of Poiseuille flow in our system. (See Fig. 2 and the discussion in Sec. II.) Presumably, the true viscosity also rises under Poiseuille flow near the channel walls, yet the variation of viscosity near the channel walls does not affect the accuracy of continuum theory in this case.

Qiao and Aluru calculated that continuum theory with the actual ion distribution from their simulations [66], eliminating the error introduced with the Poisson-Boltzmann ion density. They predicted a velocity profile more than twice what they found in simulations. However, much of this discrepancy arises because Qiao and Aluru enforced stick boundary conditions at a location far from a point that represents furthest penetration of solvent toward the walls. In our model system, applying boundary conditions at $y_{1}^{*}$ $=y_{\text {wall }}^{*}-\frac{1}{2}$ was found to produce optimal results. In our language, Qiao and Aluru enforced boundary conditions at $y_{\text {wall }}^{*}$, not $y_{1}{ }_{1}$. In other words, their continuum fluid extended into the walls to the point where solvent particles were moving over the first layer of wall particles. We will now explore the consequences of Qiao and Aluru's choice of boundary surface. The analytic theory introduced in this paper explains why their placement of the boundary too far into the wall leads to an overestimate of the velocity profile.

In Fig. 9, we present our calculations of the velocity profile using the Navier-Stokes equation [Eq. (7)] and the charge 
density for the conditions of Qiao and Aluru's case 1 simulation $[2,66]$. The charge density from Qiao and Aluru's simulations, also shown in Fig. 9, was kindly supplied to us by the authors. Three different surfaces for enforcement of stick boundary conditions are explored. Placement of the surface at $\pm 17.45 \AA$, as Qiao and Aluru have done, yields a continuum velocity profile in agreement with the one displayed in their Fig. 6, a profile that is more than twice that found in their molecular dynamics simulations. However, for solvent to penetrate as far as $\pm 17.45 \AA$, fluid particles would have to occupy the same position as wall particles. Instead, excluded volume interactions dictate that enforcement of stick boundary conditions at a smaller distance from the center of the channel is more reasonable. The two other choices for the boundary surface illustrated in Fig. 9 are physically reasonable: $\pm 15.0 \AA$, where the solvent distribution (not shown in Fig. 9) begins to rise from zero, and $\pm 14.5 \AA$, close to the peak of the solvent distribution. Setting the fluid velocity to zero at $\pm 14.5 \AA$ gives a velocity profile that peaks at almost the same value as Qiao and Aluru's simulation data. Enforcing boundary conditions at $\pm 15.0 \AA$ yields a distribution that peaks at a value roughly $16 \%$ larger. Qiao and Aluru did, in fact, notice that moving the boundary surface inward improved the agreement between continuum theory and simulations, but did not appreciate the justification for this procedure and regarded the upper velocity profile in Fig. 9 as the prediction of continuum theory. In Sec. II we justified our choice of $y_{1}^{*}$ by analyzing the Poiseuille velocity profile, and in Sec. III showed that continuum theory based on this choice could be modified to treat electro-osmotic flow. While a corresponding analysis for Qiao and Aluru's system is beyond the scope of this paper, it is clear that the choice of boundary surface for stick boundary conditions is the principal source of the discrepancy between simulation and continuum theory in their work, not failure of a spatially uniform, linear constitutive relation between shear stress and strain, as they suggest. Of course, as the channel width is pushed to smaller values there will be other situations where hydrodynamic theory will completely break down.

The analytic theory presented in Sec. III explains why placing the boundary surface too far into the wall region leads to an overestimate of the flow velocity. Our analytic theory predicts that shifting the charge density away from the boundary surface and toward the middle of the channel will increase the flow rate. This is the effect of enforcing stick boundary conditions too far from the channel center. It makes the channel width in continuum theory too wide, and places the charge away from the walls and toward the center of the channel.

Until this point, we have examined how accurately continuum theory predicts the velocity distribution using the actual charge density from simulations as input. Now we explore the consequences of using the ion distribution from Poisson-Boltzmann theory. When Poisson-Boltzmann theory with a physically reasonable channel width is used to calculate the charge density for Qiao and Aluru's system, the charge density in the center of the channel is overestimated. As predicted by the analytic theory of Sec. III, the velocity profile based on the Poisson-Boltzmann charge density is too large. It appears that Freund also finds that ions are drawn to the walls more than Poisson-Boltzmann predicts. There can be many reasons why the ion distribution in our model system deviates from Poisson-Boltzmann theory in a different direction compared to the simulations of SPC/E water, overcoming image charge repulsion to enhance the ion concentration near the walls. In both of the water studies, the Lennard-Jones well depth for the ion-wall particle interaction was the most attractive pair interaction in the system. The unique structure of the aqueous solvent could play a role in enhancing the ion concentration near the walls. Also, it is known that polarizable ions in water are attracted to an interface with a material of low dielectric constant [68-70], although this would not explain a similar discrepancy for less polarizable sodium ions. Whatever the reason, further studies are warranted for model systems, where trends are best elucidated, and also more realistic simulations.

\section{CONCLUSIONS}

From our study of a model electrolyte, the qualitative feature that emerges is that atomic level breakdown of continuum theory affects the ion distribution, but once this is corrected linear hydrodynamics provides a good description of electro-osmotic flow. In our model, the principal failure of continuum theory is neglect of the exclusion of ions from the wall region. Consequently, Poisson-Boltzmann theory underestimates the charge density in the middle of the channel. Hydrodynamics based on the unmodified Poisson-Boltzmann charge density contains an error in the driving force and, as would be expected, leads to inaccurate predictions of the flow properties. We developed a theory based on exclusion of ions from the wall region which shows that accounting for the modified ion density predicts an increased velocity profile, in agreement with our simulations. This analytic theory also predicts an important qualitative trend: Shifting the ion distribution toward the center of the channel increases the flow rate, while drawing the ions to the walls decreases the flow. This principle should be useful in the design of nanochannel devices, where chemical functionalization of the walls can be used to modify the ion distribution.

The physical origin of ion exclusion from the walls in our model is the loss of part of the solvation shell when an ion enters the fluid layer adjacent to the wall. In the wall layer, an ion loses some of the strong attractive ion-solvent interactions required to bring ions into solution, thereby creating an energy penalty for entering the wall layer. This effect was observed to varying degrees in both sets of simulation conditions we explored, and changing the ion-wall interaction would further modulate this effect. In more realistic simulations with explicit surface charge groups, the nature of ionsurface interactions will be more complex. We expect to see a range of behavior encompassing both tightly bound (Stern layer) and diffuse double-layer (Gouy layer) ions. Surface roughness is also an important factor which needs to be incorporated in a realistic treatment of electro-osmotic flow. In this work we identified a qualitative relationship between shifting of the charge density closer to or away from the walls and the overall electro-osmotic flow rate, and were able to use this relationship to understand simulation results by 
others with conditions quite different from our own. We expect that these qualitative ideas will apply in more realistic models as well.

\section{ACKNOWLEDGMENTS}

W.Z. and S.J.S. gratefully acknowledge support from the U.S. National Science Foundation Grant No. CHE-0109243. Work by A.T.C. and Z.Z. is funded by DARPA under Agreement No. F30602-00-2-0613. The authors are grateful to the contract monitors Dr. Anantha Krishnan (DARPA), Clare Thiem, and Duane Gilmour of the Air Force Research Lab (IFTC) for their support. The calculations were made possible by resource grants from the Ohio Supercomputer Center.

\section{APPENDIX: SOLUTION OF THE POISSON-BOLTZMANN EQUATIONS}

In Poisson-Boltzmann theory the average electrostatic potential $\phi(\mathbf{r})$ controls the number density of electrolyte $i$ [71],

$$
\rho_{i}(\mathbf{r})=\rho_{i}^{0} e^{-z_{i} e \phi(\mathbf{r}) / k_{B} T} .
$$

The constant $\rho_{i}^{0}$ is the density where $\phi(\mathbf{r})=0$. Poisson's equation for the electrostatic potential is

$$
\nabla^{2} \phi(\mathbf{r})=-\frac{4 \pi}{\epsilon_{r}} q(\mathbf{r}),
$$

where

$$
q(\mathbf{r})=\sum_{i} z_{i} \rho_{0, i} e^{-z_{i} e \phi(\mathbf{r}) / k_{B} T}=2 \rho_{0} \sinh \left(\frac{z e \phi(\mathbf{r})}{k_{B} T}\right) .
$$

The last equality is appropriate for a symmetrical electrolyte. The constants $\rho_{0, i}$ ( $\rho_{0}$ for a symmetrical electrolyte) are fixed by boundary conditions, as explained below. After introducing a dimensionless electrostatic potential $\psi(\mathbf{r})$,

$$
\psi(\mathbf{r})=\frac{z e}{k_{B} T} \phi(\mathbf{r})
$$

and specializing to an electrolyte confined between planar walls [26], the Poisson-Boltzmann equation becomes

$$
\frac{d^{2}}{d y^{2}} \psi(y)=\kappa^{2} \sinh [\psi(y)],
$$

where

$$
\kappa^{2}=\frac{8 \pi \rho^{0} z^{2} e^{2}}{\epsilon_{r} k_{B} T} .
$$

The solution for an electrolyte confined in a channel between parallel walls at equal electrical potentials is given in terms of Jacobi theta functions [72]:

$$
\psi(t)=\cosh ^{-1}\left[\frac{2 / k^{2}-1-\mathrm{sn}^{2}(t / k, k)}{\mathrm{cn}^{2}(t / k, k)}\right],
$$

where $t=\kappa y$,

$$
k^{2}=\frac{2}{1+\cosh \left(\psi_{0}\right)},
$$

and $\psi_{0}$ is the value of the potential in the middle of the channel. The two constants $\rho_{0}$ and $\psi_{0}$ are chosen to make the overall ionic number and charge densities equal to those of the simulations:

$$
\begin{aligned}
& \frac{1}{2 y_{0}} \int_{-y_{0}}^{y_{0}} \rho(y)=\rho_{\text {ion }} \quad \text { (overall ion density), } \\
& \frac{1}{2 y_{0}} \int_{-y_{0}}^{y_{0}} q(y)=q_{\text {ion }} \quad \text { (overall charge density). }
\end{aligned}
$$

The integrals in the two preceding equations can be expressed in terms of special functions, and the two equations solved for the two unknowns $\rho_{0}$ and $\psi_{0}$.
[1] J. B. Freund, J. Chem. Phys. 116, 2194 (2002).

[2] R. Qiao and N. R. Aluru, J. Chem. Phys. 118, 4692 (2003).

[3] H. J. C. Berendsen, J. R. Grigera, and T. P. Straatsma, J. Phys. Chem. 91, 6269 (1987).

[4] J. C. Shelley, M. Y. Shelley, R. C. Reeder, S. Bandyopadhyay, and M. L. Klein, J. Phys. Chem. B 105, 4464 (2001).

[5] C. F. Lopez, P. B. Moore, J. C. Shelley, M. Y. Shelley, and M. L. Klein, Comput. Phys. Commun. 147, 1 (2002).

[6] C. F. Lopez, S. O. Nielsen, P. B. Moore, J. C. Shelley, and M. L. Klein, J. Phys.: Condens. Matter 14, 9431 (2002).

[7] R. Goetz and R. Lipowsky, J. Chem. Phys. 108, 7397 (1998).

[8] R. Goetz, G. Gompper, and R. Lipowsky, Phys. Rev. Lett. 82, 221 (1999).

[9] J. C. Shelley and M. Y. Shelley, Curr. Opin. Colloid Interface Sci. 5, 101 (2000).

[10] B. J. Alder and T. E. Wainwright, Phys. Rev. Lett. 18, 988 (1967).
[11] B. J. Alder and T. E. Wainwright, J. Phys. Soc. Jpn. 26, 267 (1968).

[12] B. J. Alder and T. E. Wainwright, Phys. Rev. A 1, 18 (1970).

[13] A. P. Thompson, J. Chem. Phys. 119, 7503 (2003).

[14] C. Wagner, Phys. Z. 25, 474 (1924).

[15] L. Onsager and N. N. T. Samaras, J. Chem. Phys. 2, 528 (1934).

[16] E. Barouch, J. W. Perram, and E. R. Smith, Proc. R. Soc. London, Ser. A 334, 49 (1973).

[17] R. Kjellander and S. Marcelja, Chem. Phys. Lett. 112, 49 (1984); 114, 124(E) (1985).

[18] R. Kjellander and S. Marcelja, J. Chem. Phys. 82, 2122 (1985).

[19] P. Attard, D. J. Mitchell, and B. W. Ninham, J. Chem. Phys. 88, 4987 (1988).

[20] P. Attard, D. J. Mitchell, and B. W. Ninham, J. Chem. Phys. 89, 4358 (1988). 
[21] K. Meier, A. Laesecke, and S. Kabelac, Int. J. Thermophys. 22, 161 (2001).

[22] M. S. Zabaloy, J. M. V. Machado, and E. A. Macedo, Int. J. Thermophys. 22, 829 (2001).

[23] E. B. Cummings, S. K. Griffiths, R. H. Nilson, and P. H. Paul, Anal. Chem. 72, 2526 (2000).

[24] A. J. Corkhill and L. Rosenhead, Proc. R. Soc. London, Ser. A 172, 410 (1939).

[25] S. Levine and A. Suddaby, Proc. Phys. Soc., London, Sect. A 64, 287 (1951).

[26] D. Burgreen and F. R. Nakache, J. Phys. Chem. 68, 1084 (1964).

[27] C. L. Rice and R. Whitehead, J. Phys. Chem. 69, 4017 (1965).

[28] S. Levine, J. R. Marriott, and K. Robinson, J. Chem. Soc., Faraday Trans. 2 71, 1 (1975).

[29] S. Levine, J. R. Marriott, G. Neale, and N. Epstein, J. Colloid Interface Sci. 52, 136 (1975).

[30] W. Qu and D. Li, J. Colloid Interface Sci. 224, 397 (2000).

[31] A. T. Conlisk, J. McFerran, Z. Zheng, and D. Hansford, Anal. Chem. 74, 2139 (2002).

[32] W. A. Steele, The Interaction of Gases with Solid Surfaces (Pergamon, New York, 1974).

[33] V. P. Sokhan, D. Nicholson, and N. Quirke, J. Chem. Phys. 115, 3878 (2001).

[34] V. P. Sokhan, D. Nicholson, and N. Quirke, J. Chem. Phys. 117, 8531 (2002).

[35] D. J. Hansford (private communication).

[36] S. H. Behrens and D. G. Grier, J. Chem. Phys. 115, 6716 (2001).

[37] K. P. Travis and K. E. Gubbins, J. Chem. Phys. 112, 1984 (2000)

[38] D. M. Heyes, M. Barber, and J. H. R. Clark, J. Chem. Soc., Faraday Trans. 2 73, 1485 (1977).

[39] S. W. de Leeuw and J. W. Perram, Mol. Phys. 37, 1313 (1979).

[40] M. Kawata and M. Mikami, Chem. Phys. Lett. 340, 157 (2000)

[41] W. Zhu and S. J. Singer (unpublished).

[42] D. J. Evans, W. G. Hoover, B. H. Failor, B. Moran, and A. J. C. Ladd, Phys. Rev. A 28, 1016 (1983).

[43] D. J. Evans and G. P. Morriss, Statistical Mechanics of Nonequilibrium Liquids (Academic, London, 1990).

[44] F. Zhang, J. Chem. Phys. 106, 6102 (1997).

[45] J.-L. Barrat and L. Bocquet, Phys. Rev. Lett. 82, 4671 (1999).

[46] J.-L. Barrat and L. Bocquet, Faraday Discuss. 112, 119 (1999).

[47] P. A. Thompson and M. O. Robbins, Phys. Rev. A 41, 6830 (1990).

[48] C. J. Mundy, S. Balasubramanian, and M. L. Klein, Physica A 240, 305 (1997).

[49] M. J. Stevens, M. Mondello, G. S. Grest, S. T. Cui, H. D.
Cochran, and P. T. Cummings, J. Chem. Phys. 106, 7303 (1997).

[50] J. Koplik, J. R. Banavar, and J. F. Willemsen, Phys. Fluids A 1, 781 (1989).

[51] R. F. Cracknell, D. Nicholson, and N. Quirke, Phys. Rev. Lett. 74, 2463 (1995).

[52] S. K. Bhatia and D. Nicholson, Phys. Rev. Lett. 90, 016105 (2003)

[53] S. K. Bhatia and D. Nicholson, J. Chem. Phys. 119, 1719 (2003).

[54] M. Cieplak, J. Koplik, and J. R. Banavar, Phys. Rev. Lett. 86, 803 (2001).

[55] G. Arya, H.-C. Chang, and E. J. Maginn, Mol. Simul. 29, 697 (2003).

[56] K. P. Travis, B. D. Todd, and D. J. Evans, Physica A 240, 315 (1997).

[57] P. A. Thompson and S. M. Troian, Nature (London) 389, 360 (1997).

[58] T. M. Galea and P. Attard, Langmuir 20, 3477 (2004).

[59] J. Gao, W. Luedtke, and U. Landman, Tribol. Lett. 9, 3 (2000).

[60] I. Bitsanis, J. J. Magda, M. Tirrell, and H. T. Davis, J. Chem. Phys. 87, 1733 (1987).

[61] I. Bitsanis, T. K. Vanderlick, M. Tirrell, and H. T. Davis, J. Chem. Phys. 89, 3152 (1988).

[62] T. A. Desai, D. J. Hansford, L. Kulinsky, A. H. Nashat, G. Rasi, J. Tu, Y. Wang, M. Zhang, and M. Ferrari, Biomed. Microdevices 2, 11 (1999).

[63] D. Hansford, T. Desai, and M. Ferrari, in Biochip Technologies, edited by L. Kricka and J. Cheng (Harwood Academic, Chum, Switzerland, 2001), p. 341.

[64] C. Lee, E.-H. Yang, N. V. Myung, and T. George, Nano Lett. 3, 1339 (2003).

[65] C. Lee, E.-H. Yang, N. V. Myung, and T. George, in Seventh International Conference on Miniaturized Chemical and Biochemical Analysis Systems, 2003 (unpublished), p. 643.

[66] See Fig. 6 of Ref. [2].

[67] S. Balasubramanian, C. J. Mundy, and M. L. Klein, J. Chem. Phys. 105, 11190 (1996).

[68] E. M. Knipping, M. J. Lakin, K. L. Foster, P. Jungwirth, D. J. Tobias, R. B. Gerber, D. Dabdub, and B. J. Finlayson-Pitts, Science 288, 301 (2001).

[69] P. Jungwirth and D. J. Tobias, J. Phys. Chem. B 104, 7702 (2000).

[70] P. Jungwirth and D. J. Tobias, J. Phys. Chem. B 106, 6361 (2002).

[71] J. Israelachvili, Intermolecular and Surface Forces, 2nd ed. (Academic, San Diego, 1991).

[72] Handbook of Mathematical Functions, Nat. Bur. Stand. Appl. Math. Ser. No. 55, edited by M. Abramowitz and I. A. Stegun (U.S. GPO, Washington, D.C., 1972). 